\title{
Restricted Component Additive Games
}

\author{
IMMa CURIEL
}

Department of Mathematics and Statistics, University of Maryland Baltimore County, MD 21228, USA

\author{
Herbert Hamers and Stef TiJs \\ Center and Department of Econometrics, Tilburg University, P.O. Box 90153, 5000 LE Tilburg, \\ The Netherlands
}

Jos POTTERS

Department of Mathematics, University of Nijmegen, Toernooiveld, 6525 ED Nijmegen, The Netherlands

Abstract: Curiel, Potters, Prasad, Tijs and Veltman (1993) introduced component additive games which are cooperative TU games that arise by imposing an order on the player set. Further, they introduced the $\beta$-rule which allocates a core element to each component additive game.

In this paper we consider the class of restricted component additive games that arise by restricting the attention to head-tail coalitions. The extreme points of the corresponding restricted core are characterized. Further, it is shown that the $\beta$-rule is the barycenter of the corresponding restricted core and that the $\beta$-rule coincides with the nucleolus of this restricted game.

\section{Introduction}

Component additive games are first introduced in Curiel, Potters, Prasad, Tijs and Veltman (1993). For these games Curiel, Potters, Prasad, Tijs and Veltman (1994) showed that the $\beta$-rule, which is the average of the marginal vector of payoffs described by $\sigma$ and the marginal vector described by $\sigma^{-1}$, is a coreelement of the corresponding $\sigma$ component additive game. Potters and Reijnierse (1993) showed that for a particular class of games, that contains the class of component additive games, the bargaining set coincides with its core and the kernel consists of the nucleolus only.

Restricted games are cooperative games where the collection of feasible coalitions is restricted. Myerson (1977) and Owen (1986) considered restricted games that arose from communication situations. Faigle (1989) studied the core of a restricted game. He gives a generalization for the well-known Shapley-Bondareva theorem. Kuipers (1994) introduced a nucleolus for restricted games. This restricted nucleolus is an example of the general nucleolus for TU games introduced by Maschler, Potters and Tijs (1992). 
This paper focuses on restricted games of component additive games that arise from the family of head-tail coalitions. This family, denoted by $\mathscr{H}$, consists of the coalitions $\{1, \ldots, i\}$ or $\{i+1, \ldots, n\}, 1 \leq i \leq n-1$ if the given order $\sigma$ is $1 \prec 2 \prec \cdots \prec n$. There are several arguments to consider restricted component additive game. For component additive games the nucleolus can be calculated easily, in fact the $\beta$-rule coincides with the nucleolus. This implies for the class of sequencing games (cf. Curiel, Pederzoli and Tijs (1989)), which is a subclass of component additive games, that the solution concept called the Equal Gain Splitting rule can be characterized as the nucleolus of the corresponding restricted component additive games. Further, restricted component additive games can be seen as an example of restricted games with a bounded core (cf. Derks and Reijnierse (1993)).

For the class of head-tail restricted games we characterize the extreme points of the corresponding restricted core. Further, it is shown that the $\beta$-rule is in the barycenter of the corresponding restricted core. The main result will be that the $\beta$-value coincide with the nucleolus of this restricted game.

\section{Component Additive Games}

This section describes the class of $\sigma$-component additive games and recalls the definition of the $\beta$-rule. Further, we consider the class of restricted games corresponding to the family of head-tail coalitions.

A cooperative game is a pair $(N, v)$ where $N$ is a finite set of players and $v$ is a mapping $v: 2^{N} \rightarrow \mathbf{R}$ with $v(\varnothing) \neq 0$ and where $2^{N}$ is the collection of all subsets of $N$. A game $(N, v)$ is called superadditive if for all coalitions $S, T \in 2^{N}$ with $S \cap T=\varnothing$ we have $v(S \cup T) \geq v(S)+v(T)$.

Let $N=\{1, \ldots, n\}$ be the player set and let $\sigma$ be a permutation of $N$ that defines an order on $N$. Then a coalition $S \subset N$ is called connected with respect to $\sigma$ if for all $i, j \in S$ and $k \in N, \sigma(i)<\sigma(k)<\sigma(j)$ implies $k \in S$. Let $S \subset N$ be a coalition that is not connected. Then a coalition $T \subset S$ is called a component of $S$ if $T$ is connected and for any $i \in S \backslash T, T \cup\{i\}$ is not connected. The set of components form a partition of $S$ and is denoted by $S \backslash \sigma$. For example if $\sigma$ is the order $1 \prec 2 \prec 3 \prec 4 \prec 5$ and $S=\{1,2,4,5\}$ then $S \backslash \sigma=\{\{1,2\},\{4,5\}\}$.

A cooperative game $(N, v)$ is called $\sigma$-component additive w.r.t. the permutation $\sigma$ if this game satisfies the following two conditions:

(a) $v$ is superadditive

(b) $v(S)=\sum_{T \in S \backslash \sigma} v(T)$.

Let $(N, v)$ be a $\sigma$-component additive game then the $\beta$-rule is defined for all $i \in N$ by 


$$
\beta_{i}(v)=\frac{1}{2}\{v(P(\sigma, i) \cup\{i\})-v(P(\sigma, i))+v(F(\sigma, i) \cup\{i\})-v(F(\sigma, i))\}
$$

where $P(\sigma, i)=\{j \mid \sigma(j)<\sigma(i)\}$ is the set of predecessors of $i$ and $F(\sigma, i)=$ $\{k \mid \sigma(k)>\sigma(i)\}$ the set of followers of $i$. Curiel et al. (1994) showed that the $\beta$-rule assigns to each $\sigma$-component additive game a vector that is in the core of this game.

Example 1: Consider the $\sigma$-component additive game $(N, v)$ where $N=\{1,2,3\}$ and $\sigma$ is the order $1 \prec 2 \prec 3$. Let $v(\{i\})=0$ for all $i \in N, v(\{1,2\})=4$, $v(\{2,3\})=4$ and $v(N)=14$. Since $(N, v)$ is $\sigma$-component additive we have that $v(\{1,3\})=v(\{1\})+v(\{3\})=0$. Further, $\beta=(5,4,5)$.

\section{The Results}

This section gives the definition of a head-tail restricted game and its core. We will describe the extreme point of the core of a head-tail restricted game and show that the $\beta$-rule is the barycenter of the corresponding core. Finally, we show that the $\beta$-rule coincides with the nucleolus of the restricted head-tail game.

In the following we will assume that $\sigma$ is the order $1 \prec 2 \prec \cdots \prec n$. For notational convenience we will omit the prescript $\sigma$.

The set of head-tail coalitions with respect to $\sigma$ is defined by

$$
\mathscr{H}:=\{\{1, \ldots, i\},\{i+1, \ldots, n\} \mid 1 \leq i \leq n-1\} .
$$

The restricted head-tail game $(\mathscr{H}, v)$ of a component additive game $(N, w)$ is defined by $v(S)=w(S)$ for all $S \in \mathscr{H}$. The core of the restricted game $(N, w)$ is defined by

$$
C^{\mathscr{H}}(v):=\left\{x \in \mathbf{R}^{n} \mid x(S) \geq v(S) \text { for all } S \in \mathscr{H} \text { and } x(N)=v(N)\right\}
$$

where $x(S)=\sum_{i \in S} x_{i}$. Obviously, we have that the core $C(w)$ of the component additive game $(N, w)$ is contained in the head-tail core $C^{\mathscr{H}}(v)$ of the corresponding restricted head-tail game $(N, v)$.

Example 2: Consider the component additive game of example 1. An extreme point of $C^{\mathscr{H}}(v)$ is the vector $(10,-6,10)$. Obviously, this vector is not an ele- 


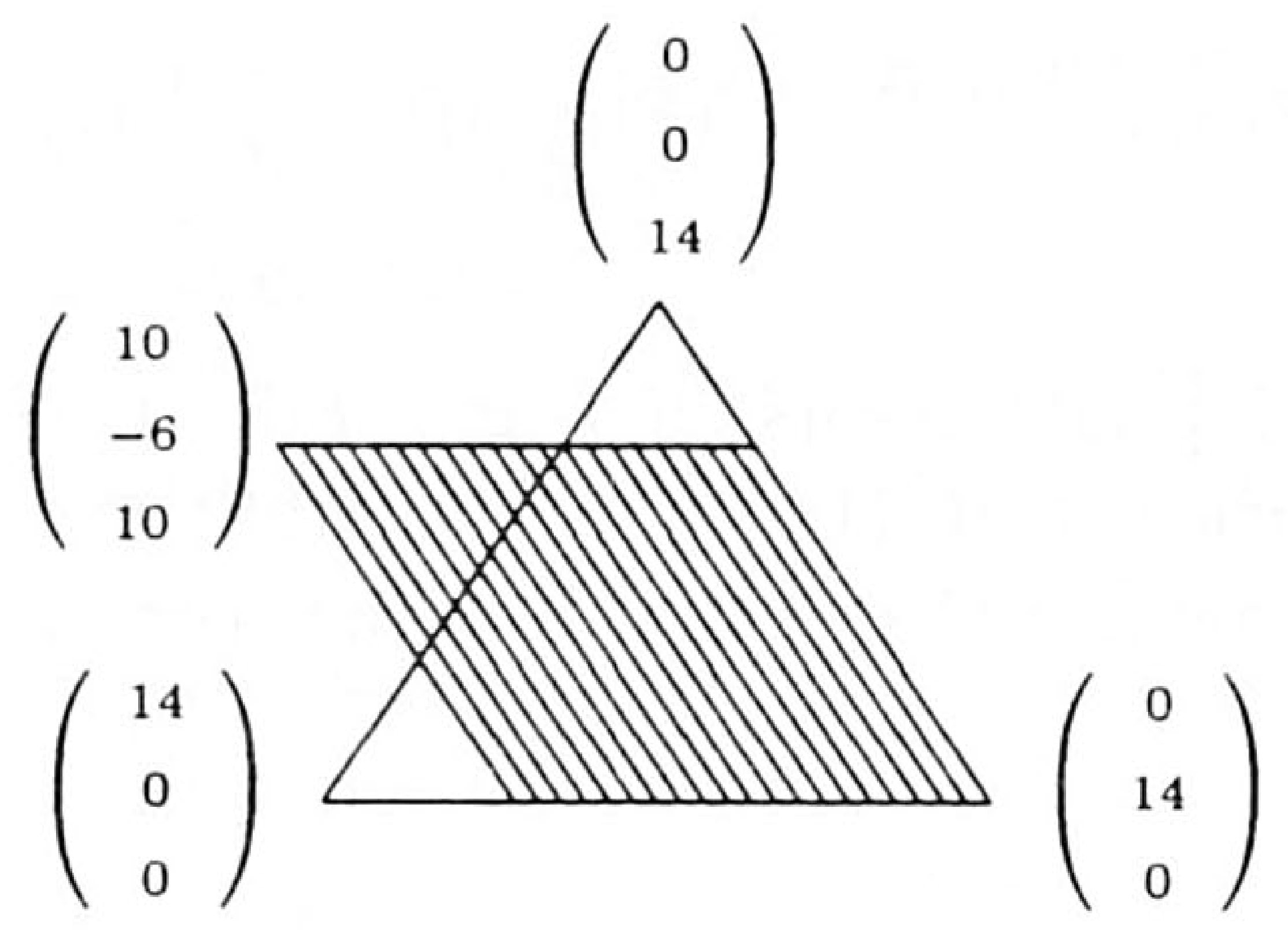

Fig. 1. The shaded area represents the head-tail core

ment of $C(v)$ (see figure 1), which means that the core can be a proper subset of the head-tail core.

The dual game $\left(N, v^{*}\right)$ of $(N, v)$ is defined by $v^{*}(S)=v(N)-v\left(S^{c}\right)$ for all $S \subset N$. Note that $v(N)=v^{*}(N)$ and that $v(S) \leq v^{*}(S)$ for all $S$ by the superadditivity of $(N, v)$.

Now (2) is equivalent to

$$
\begin{aligned}
C^{\mathscr{H}}(v) & =\left\{x \in \mathbf{R}^{n} \mid v(\{1, \ldots, i\}) \leq \sum_{k=1}^{i} x_{k} \leq v^{*}(\{1, \ldots, i\}) \text { for all } i \in N\right\} \\
& =\left\{x \in \mathbf{R}^{n} \mid x=L^{-1} y, v(\{1, \ldots, i\}) \leq y_{i} \leq v^{*}(\{1, \ldots, i\}) \text { for all } i \in N\right\}
\end{aligned}
$$

where $L$ is the $n \times n$ non-singular lower triangular matrix with ones on and below the diagonal, and zeros above the diagonal. It is easy to see that the linear map $L$ gives a $1-1$ correspondence between $C^{\mathscr{H}}(v)$ and the set $D$ defined by

$$
D:=\left\{y \in \mathbf{R}^{n} \mid v(\{1, \ldots, i\}) \leq y_{i} \leq v^{*}(\{1, \ldots, i\}) \text { for all } i \in N\right\}
$$

Consequently, there is a 1-1 correspondence between the extreme points of both sets. Clearly, the extreme points of $D$ correspond to a system of $n$ equations of the form

$$
y_{i}=a_{i}, \quad i=1, \ldots, n \quad \text { where } a_{i} \in\left\{v(\{1, \ldots, i\}), v^{*}(\{1, \ldots, i\})\right\} \quad \text { for all } i \in N \text {. }
$$

Let $J \subset N$. We put $h^{J}=L^{-1}\left(y^{J}\right)$ where $y^{J}$ is the extreme point of $D$ corresponding to the system of equations given by

$$
y_{i}^{J}= \begin{cases}v(\{1, \ldots, i\}) & i \in N \backslash J \\ v^{*}(\{1, \ldots, i\}) & i \in J\end{cases}
$$


Then $E=\left\{h^{J} \mid J \subset N\right\}$ is the set of extreme points of $C^{\mathscr{H}}(v)$. Consequently, we have

Theorem 1: $C^{\mathscr{H}}(v)=\operatorname{conv}\left\{h^{J} \mid h^{J} \in E\right\}$ where $h^{J}$ corresponds to the solutions of the set of equations

$$
\begin{aligned}
& \sum_{k=1}^{i} x_{k}=v^{*}(\{1, \ldots, i\}) \quad i \in J \\
& \sum_{k=1}^{i} x_{k}=v(\{1, \ldots, i\}) \quad i \in N \backslash J
\end{aligned}
$$

The number of extreme points is $2^{|I|}$ where $I=\{i \in N \mid v(\{1, \ldots, i\}) \neq$ $\left.v^{*}(\{1, \ldots, i\})\right\}$. Hence, in the generic case we have $2^{n-1}$ different extreme points since $v^{*}(N)=v(N)$ and consequently $n \notin I$.

The following theorem shows that the average of the extreme points of the head tail core generated by (3) is the $\beta$-rule.

Theorem 2: Let $(N, v)$ be a component additive game. Then $\beta(v)=\frac{1}{2^{n-1}} \sum_{J \subset N} h^{J}$.

Proof: Note that for all $i \in N$ and all $J \subset N$ we have $y_{i}^{J}+y_{i}^{N \backslash J}=$ $v(\{1, \ldots, i\})+v^{*}(\{1, \ldots, i\})$. Consequently, for all $J \subset N$ it follows that $L\left(h^{J}+h^{N \backslash J}\right)=y^{J}+y^{N \backslash J}=y^{\varnothing}+y^{N}=L\left(h^{\varnothing}+h^{N}\right)$. Since $h^{\varnothing}+h^{N}=2 \beta(v)$ we have

$$
\frac{1}{2^{n-1}} \sum_{J \subset N} h^{J}=\frac{1}{2^{n}} \sum_{J \subset N}\left(h^{J}+h^{N \backslash J}\right)=\frac{1}{2^{n}} 2^{n} \beta(v)=\beta(v) .
$$

The nucleolus of a restricted game is introduced in Kuipers (1994). Next, we will recall the definition of this nucleolus with respect to restricted head-tail games. Let $F:=\left(F_{T}\right)_{T \in \mathscr{H}}$, where $F_{T}$ are the excess functions defined by $F_{T}(x):=$ $v(T)-x(T)$. The function $\Theta: \mathbf{R}^{\mathscr{H}} \rightarrow \mathbf{R}^{|\mathscr{H}|}$ is the map that orders the coordinates in a weakly decreasing order. Then the restricted nucleolus is defined by

$$
\mathscr{N}(\mathscr{H}, v)=\left\{x \in \Pi \mid \Theta \circ F(x) \preceq_{L} \Theta \circ F(y) \text { for all } y \in \Pi\right\}
$$

where $\Pi=\left\{x \in \mathbf{R}^{n} \mid x(N)=v(N)\right\}$. Note that the restricted nucleolus is an example of the general nucleolus defined on the class of TU games (cf. Maschler, Potters, Tijs (1992)). We need the following lemma. 
Lemma 1: Let $(N, v)$ be a component additive game. Then $\beta(v)$ is the unique solution of the system of equations given by

$$
x(S)-v(S)=x\left(S^{c}\right)-v\left(S^{c}\right) \quad \text { for all } S \in \mathscr{H} \cup N .
$$

Proof: For $k \in N \backslash\{n\}$ we have

$$
x(\{1, \ldots, k\})-v(\{1, \ldots, k\})=x(\{k+1, \ldots, n\})-v(\{k+1, \ldots, n\})
$$

Let $m \in\{1, \ldots, n-2\}$ and substitute $k=m$ and $k=m+1$ in equation (4), respectively. Subtraction of these two equations yields

$$
\begin{aligned}
& x(\{m+1\})-v(\{1, \ldots, m\})+v(\{1, \ldots, m+1\}) \\
& =-x(\{m+1\})-v(\{m+1, \ldots, n\})-v(\{m+2, \ldots, n\}) .
\end{aligned}
$$

Hence, $x(\{m+1\})=\beta_{m+1}(v)$ for all $m+1 \in\{2, \ldots, n-1\}$. From equation $x(N)=v(N)$ and equation (4) with $k=1(k=n)$ it follows that $x(\{1\})=$ $\beta_{1}(v)\left(x(\{n\})=\beta_{n}(v)\right)$.

The following theorem states that the $\beta$-rule of a component additive game $(N, w)$ coincides with the restricted nucleolus of the corresponding restricted head-tail game.

Theorem 3: Let $(N, w)$ be a component additive game and let $(N, v)$ be the corresponding restricted head-tail game. Then the $\beta$-rule of $(N, w)$ is the unique element of $\mathscr{N}(\mathscr{H}, v)$.

Proof: First it is shown that $\mathscr{N}(\mathscr{H}, v)$ is a non-empty set. Let $y \in \Pi \backslash C^{\mathscr{H}}(v)$, then there exists an $S \in \mathscr{H}$ such that $y(S)<v(S)$. Since for any $x \in C^{\mathscr{H}}(v)$ it holds that $x(T) \geq v(T)$ for all $T \in \mathscr{H}$ we have that $\Theta \circ F(x) \prec_{L} \Theta \circ F(y)$. Hence, $\mathscr{N}(\mathscr{H}, v)=\left\{x \in C^{\mathscr{H}} \mid \Theta \circ F(x) \preceq_{L} \Theta \circ F(y)\right.$ for all $\left.y \in C^{\mathscr{H}}(v)\right\}$. Since $C^{\mathscr{H}}(v)$ is a compact set and $\Theta \circ F$ is a continuous map it follows that $\mathscr{N}(\mathscr{H}, v) \neq \varnothing$.

Let $x \in \mathscr{N}(\mathscr{H}, v)$. Suppose there exists a player $k \in N$ and a number $\varepsilon>0$ such that

$$
[v(\{1, \ldots, k\})-x(\{1, \ldots, k\})]-[v(\{k+1, \ldots, n\})-x(\{k+1, \ldots, n\})]>\varepsilon
$$


Take $y \in \Pi$ such that $y_{i}=x_{i}$ for all $i \in N \backslash\{k, k+1\}, y_{k}=x_{k}+\varepsilon$ and $y_{k+1}=$ $x_{k+1}-\varepsilon$. Then

$$
\begin{aligned}
& v(T)-x(T)=v(T)-y(T) \quad \text { for } T \in \mathscr{H} \backslash\{\{1, \ldots, k\},\{k+1, \ldots, n\}\}, \\
& v(\{1, \ldots, k\})-x(\{1, \ldots, k\})=v(\{1, \ldots, k\})-y(\{1, \ldots, k\})+\varepsilon
\end{aligned}
$$

and

$$
\begin{aligned}
& v(\{k+1, \ldots, n\})-x(\{k+1, \ldots, n\}) \\
& \quad=v(\{k+1, \ldots, n\})-y(\{k+1, \ldots, n\})-\varepsilon .
\end{aligned}
$$

Hence, $\Theta \circ F(y) \prec_{L} \Theta \circ F(x)$. This is in contradiction with the definition of $\mathscr{N}(\mathscr{H}, v)$. In a similar way we can show that there exists no $k \in N$ such that

$$
\begin{aligned}
& {[v(\{1, \ldots, k\})-x(\{1, \ldots, k\})]-[v(\{k+1, \ldots, n\})-x(\{k+1, \ldots, n\})]} \\
& \quad<0 .
\end{aligned}
$$

This implies that for all $S \in \mathscr{H}$ we have $F_{S}(x)=F_{S^{c}}(x)$. Then lemma 1 implies that $\{\beta(v)\}=\mathscr{N}(\mathscr{H}, v)$.

\section{References}

Curiel I, Pederzoli G, Tijs S (1989) Sequencing games. European Journal of Operational Research 40:344-351

Curiel I, Potters J, Rajendra Prasad V, Tijs S, Veltman B (1993) Cooperation in one machine scheduling. Zeitschrift für Operations Research 38:113-129

Curiel I, Potters J, Rajendra Prasad V, Tijs S, Veltman B (1994) Sequencing and cooperation. Operations Research 42:566-568

Derks J, Reijnierse H (1993) On the core of a collection of coalitions. Report M93-12, RL Maastricht, The Netherlands

Faigle U (1989) Cores of games with restricted cooperation. Zeitschrift für Operations Research $33: 405-422$

Kuipers J (1994) Combinatorial methods in cooperative game theory. Ph.D. Dissertation, Maastricht

Maschler M, Potters J, Tijs S (1992) The general nucleolus and the reduced game property. International Journal of Game Theory 21:85-106 
Myerson R (1977) Graphs and cooperation in games. Mathematics of Operations Research 2:224229

Owen G (1986) Values of graph-restricted games. SIAM Journal of Algebraic and Discrete Models $7: 210-220$

Potters J, Reijnierse H (1995) is component additive games. International Journal of Game Theory $24: 49-56$

Received: February 1995

Revised version received: March 1996 